\title{
Newborns exposed to opioids need mothers more than NICU, say pediatricians
}

\author{
— Cite as: CMAJ 2018 January 29;190:E123. doi: 10.1503/cmaj.109-5550
}

Posted on cmajnews.com on Jan. 11, 2018.

A $\mathrm{n}$ increasing number of babies are being born already exposed to opioids, and separating them from their mothers for treatment may be making a bad situation worse, the Canadian Paediatric Society (CPS) warned in a new guidance document.

Up to $75 \%$ of babies born to mothers taking opioids will require treatment for withdrawal symptoms, also known as neonatal abstinence syndrome or NAS. About 1850 babies born in Canada last year had the condition, up 27\% since 2012-13.

Usually, these babies are whisked away to a neonatal intensive care unit, or NICU, for treatment with decreasing doses of morphine, while their mothers return home. But according to CPS, neither the NICU nor the morphine may be necessary. And the weeks of separation involved in treatment can be harmful to early bonding and attachment.

"We need to look at this differently," said Dr. Thierry Lacaze, chair of the CPS Fetus and Newborn Committee. "These are not intensive-care babies and most of the time breastfeeding is an issue because of the separation." In rural areas, long distances to the nearest NICU mean "the separation is even worse."

CPS recommends keeping opioiddependent mothers and babies together from birth, and focusing on non-drug methods of reducing the discomfort of withdrawal. These include breastfeeding, skin-to-skin contact, swaddling, reducing noise, lowering lights, and limiting stimulation. "We believe this kind of program will dramatically impact both short- and long-term outcomes," said Lacaze.

This type of "rooming-in" program has been shown to increase bonding and

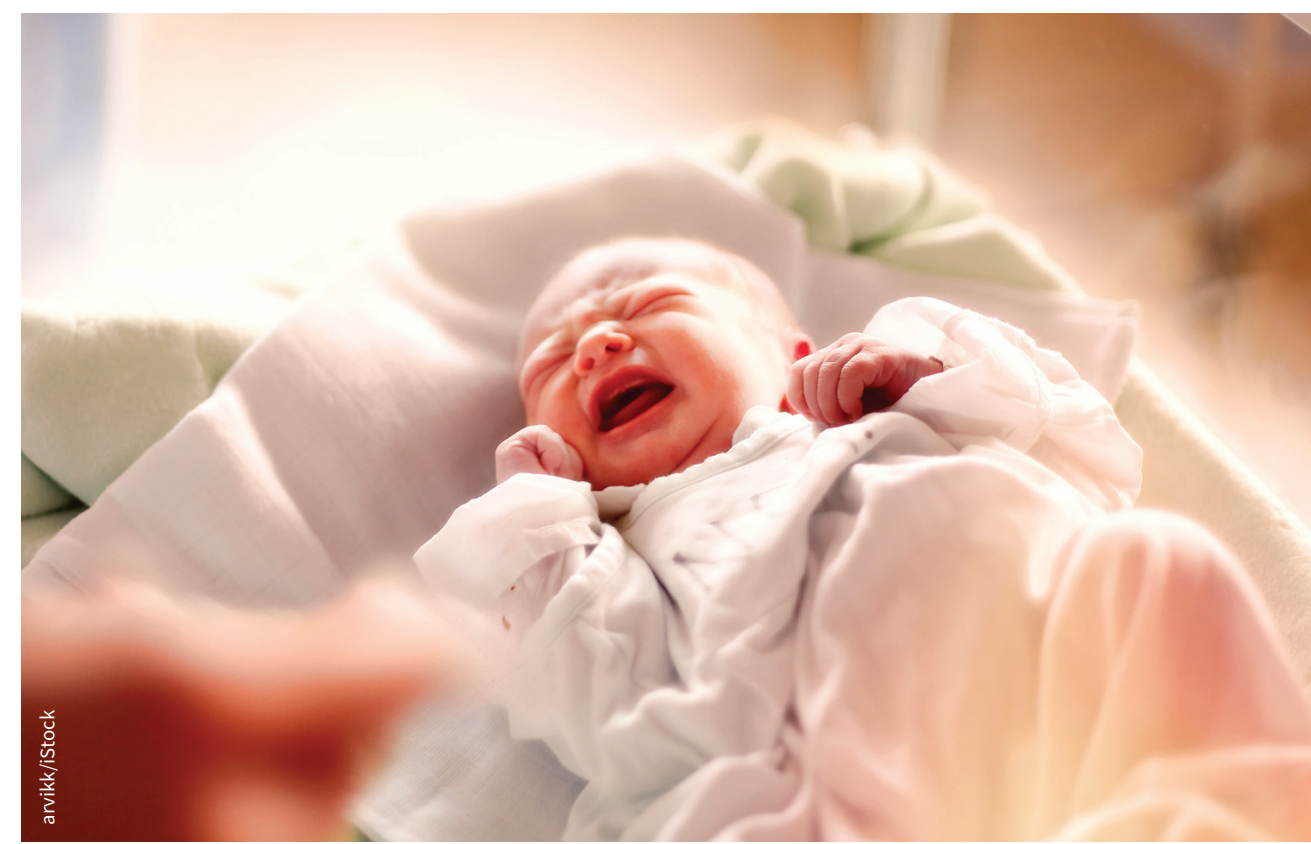

Keeping opioid-dependent mothers and babies together from birth improves health outcomes, says the Canadian Paediatric Society.

breastfeeding rates, and reduce hospital stays, NICU admissions and the use of drugs like morphine in treatment for NAS. Breastfeeding in particular reduces the need for morphine, "the idea being babies get a small amount of opioid through the breast milk instead," said Lacaze.

The approach "sounds easy on paper," he said. But in practice, obstetric staff are often uncomfortable handling these cases. Babies with NAS have problems with sleeping, feeding and irritability. They have a persistent, high-pitch cry, and can suffer seizures. In short, "these babies require a lot of attention," and staff who are confident in handling their care.

It's also challenging to secure the rooms and budget to keep women and babies on obstetric wards for weeks instead of days.
Although cheaper than providing care in the NICU, "it's sometimes difficult for the policy people to understand you need to spend money today to save a lot of money tomorrow," said Lacaze.

The tendency to shame and blame women who use opioids while pregnant complicates these challenges, Lacaze added. "For a long time, there's been stigma, and those women have a very strong guilt, so you need to get away from that."

Hospitals in British Columbia, Alberta, Ontario and Quebec now have rooming-in programs underway. "I think in the next few years, it's going to become the standard of care," said Lacaze.

Lauren Vogel, CMAJ 